\title{
A meta-analysis of trait differences between invasive and non-invasive plant species
}

\author{
Mark van Kleunen, ${ }^{*}$ Ewald \\ Weber ${ }^{1}$ and Markus Fischer \\ Institute of Plant Sciences and \\ Oeschger Centre, University of \\ Bern, Altenbergrain 21, $\mathrm{CH} 3013$ \\ Bern, Switzerland \\ ${ }^{1}$ Present address: Institute of \\ Biochemistry and Biology, \\ Biodiversity Research, University \\ of Potsdam, Maulbeerallee 1, \\ 14469 Potsdam, Germany. \\ *Correspondence: $E$ mail: \\ vkleunen@ips.unibe.ch
}

\begin{abstract}
A major aim in ecology is identifying determinants of invasiveness. We performed a meta analysis of 117 field or experimental garden studies that measured pair wise trait differences of a total of 125 invasive and 196 non invasive plant species in the invasive range of the invasive species. We tested whether invasiveness is associated with performance related traits (physiology, leaf area allocation, shoot allocation, growth rate, size and fitness), and whether such associations depend on type of study and on biogeographical or biological factors. Overall, invasive species had significantly higher values than non invasive species for all six trait categories. More trait differences were significant for invasive vs. native comparisons than for invasive vs. non invasive alien comparisons. Moreover, for comparisons between invasive species and native species that themselves are invasive elsewhere, no trait differences were significant. Differences in physiology and growth rate were larger in tropical regions than in temperate regions. Trait differences did not depend on whether the invasive alien species originates from Europe, nor did they depend on the test environment. We conclude that invasive alien species had higher values for those traits related to performance than non invasive species. This suggests that it might become possible to predict future plant invasions from species traits.
\end{abstract}

\section{Keywords}

Comparative studies, exotic species, functional traits, invasiveness, literature review, meta analysis, non indigenous species, non native species.

\section{INTRODUCTION}

Owing to the increasing influence of humans, many species have invaded and continue to invade new regions at an unprecedented rate, exerting strong impacts on ecosystems and human welfare (Mooney et al. 2005; Pimentel et al. 2005). Hence, causes of invasiveness of alien plant species are an important research topic in ecology and invasion biology, and of considerable applied relevance. It is very likely that functional traits of species, such as the ones related to physiology, biomass allocation, growth rate, size and fitness, promote invasiveness (Kolar \& Lodge 2001; Grotkopp et al. 2002, 2004; van Kleunen \& Richardson 2007), but progress in the search for traits conferring invasiveness has been slow. One reason for this could be that with few exceptions (e.g. Lake \& Leishman 2004;
Hamilton et al. 2005; Colautti et al. 2006; Leishman et al. 2007; Mason et al. 2008) most studies of species traits associated with invasiveness (reviewed in Pyšek \& Richard son 2007) have been restricted to relatively simple traits, such as plant height and growth form, that are readily available for large numbers of species from databases, such as floral compendia. Many potentially important functional traits for invasiveness, however, have to be measured on invasive and non invasive species grown under common environmental conditions.

Most comparative experimental studies testing for traits associated with invasiveness involve only small numbers of species, which precludes broader generalization of their results. Narrative literature reviews have, however, provided some insights into the generality of the observed trait differences reported in these studies. Daehler (2003) 
reviewed 119 studies, mainly experimental ones, comparing traits between invasive alien plant species and native plant species, and Pyšek \& Richardson (2007) reviewed 64 studies comparing confamilial (and congeneric) invasive alien plant species and non invasive, both native and alien, species. These two reviews found that some characteristics, such as high fecundity, are frequently associated with invasiveness, but they also found some contradictory patterns. For example, Pyšek \& Richardson (2007) found an association of fast growth rate with invasiveness, whereas Daehler (2003) did not find such a pattern. Both reviews simply counted the number of studies that found significantly higher trait values for invasive than for non invasive species, the ones that found significantly lower values for invasive than for non invasive species and the number of studies that did not find significant differences. However, this vote counting approach is overly conservative, has low statistical power, and does not consider the magnitude of the differences between invasive and non invasive species (Rosenberg $\mathrm{et}$ al. 2000). A more powerful approach to test for general patterns across multiple studies, which accounts for differences in sample sizes and the magnitude of the differences, is meta analysis (Hedges \& Olkin 1985; Rosenberg et al. 2000).

We calculated effect sizes (Hedges' $d$ ) and their variances for differences in trait values between invasive alien plant species and non invasive, either native or alien, plant species from 117 published studies. In total, these studies included data of 125 invasive and 196 non invasive plant species. In these studies, traits of the invasive and non invasive species have been measured mostly in experimental garden settings. We used meta analysis to assess whether invasive species and non invasive species differ in traits related to physiol ogy, leaf area allocation, shoot allocation, growth rate, size and fitness (Table 1). Because traits within and between trait categories are frequently causally related (e.g. Grotkopp et al. 2002, 2004) or associated through trade offs (e.g. Westoby et al. 2002), we expected that invasive and non invasive species can differ in any of these trait categories. Because growth rate, size and fitness are most closely associated with plant performance, we expected traits of these categories to be most consistently associated with invasiveness (Grotkopp et al. 2002; Rejmánek et al. 2005; Mason et al. 2008). However, a previous meta analysis comparing differences in size and reproduction between invasive alien species and native species from 15 studies found that both groups of species did not differ signifi cantly in size and that invasive species had a lower reproductive allocation than native species (Hawkes 2007). In comparison with this previous meta analysis, we included many more studies, species and species traits, providing therefore a more powerful data set.

There are several factors related to the design of studies that might affect the outcomes of comparisons between
Table 1 Categories of traits and examples of corresponding traits as obtained from the publications. When a low value of a trait indicates biologically the same as high values of other traits in the same trait category (e.g. a low root weight ratio indicates a high shoot allocation), we changed the sign of the effect sizes of this trait (-)

\begin{tabular}{|c|c|}
\hline Trait category & Traits \\
\hline Physiology & $\begin{array}{l}\text { Photosynthetic rate, transpiration, leaf } \\
\text { construction costs }(-) \text {, tissue nitrogen } \\
\text { content, nitrogen use efficiency, water } \\
\text { use efficiency }\end{array}$ \\
\hline $\begin{array}{l}\text { Leaf area } \\
\text { allocation }\end{array}$ & $\begin{array}{l}\text { Leaf area index, leaf area ratio, specific } \\
\text { leaf area, leaf mass ratio, specific leaf } \\
\text { mass }(-)\end{array}$ \\
\hline Shoot allocation & $\begin{array}{l}\text { Shoot root ratio, root fraction }(-) \text {, } \\
\text { root weight ratio }(-) \text {, root shoot ratio }(-)\end{array}$ \\
\hline Growth rate & Increase in size or biomass over time \\
\hline Size & $\begin{array}{l}\text { Biomass of roots, shoots and complete } \\
\text { plants, plant height, total leaf area }\end{array}$ \\
\hline Fitness & $\begin{array}{l}\text { All characters measuring number of flowers } \\
\text { or seeds per plant, per flower head, per } \\
\text { inflorescence, per fruit, all characters } \\
\text { associated with seed germination, all traits } \\
\text { associated with survival [stem survival, } \\
\text { seedling establishment, mortality ( ), } \\
\text { survival time] }\end{array}$ \\
\hline
\end{tabular}

invasive alien species and non invasive species. First, although both types of comparisons (invasive alien species vs. native species, and invasive alien species vs. non invasive alien species) test for traits associated with invasiveness, their exact interpretations are different. Invasive alien vs. native comparisons address the question what traits of invading species enhance their potential to increase over native species, and invasive alien vs. non invasive alien comparisons address the question what traits distinguish successful invaders from alien species that have not invaded successfully (Hamilton et al. 2005). Therefore, we tested whether mean effect sizes of trait differences depend on whether the invasive alien species is compared to a native species or to a non invasive alien species. Second, some of the native species might be invasive elsewhere, which would imply that some studies compared invasive alien species to other invasive alien species (Rejmánek 1999; Muth \& Pigliucci 2006), at least at a global scale. Therefore, we also tested whether mean effect sizes of trait differences depend on whether the native species is known to be invasive elsewhere. Third, because trait differences between species may strongly depend on taxonomic relatedness (Daehler 1998; Pyšek 1998; van Kleunen et al. 2007) and similarity in growth form of the species (Bucharova \& van Kleunen 2009), we also tested whether mean effect sizes of trait 
differences depend on whether con familial species, and on whether species with the same growth form, were com pared.

Differences in traits between invasive and non invasive species might also depend on biogeographical and biological factors. First, because different types of traits may be important under different climatic conditions (Pyšek \& Richardson 2006), we tested whether mean effect sizes of trait differences depend on the climate of the study region (temperate vs. tropical or subtropical). Second, it has frequently been suggested that European species are better invaders than non European species due to their longer evolutionary history under anthropogenic disturbance (e.g. Gray 1879; La Sorte et al. 2007). Therefore, we tested whether mean effect sizes of trait differences depend on whether the invasive species is native to Europe. Finally, it has been noted that trait differences between invasive alien species and non invasive species might depend on the test environment (Daehler 2003; Richards et al. 2006). There fore, we also tested for the subset of studies that included more than one environment, whether mean effect sizes of trait differences depend on the quality of the environment.

This meta analysis allows us to test for general patterns across multiple studies and a wide range of plant species. Moreover, it allows us to identify gaps in general knowledge on determinants of plant invasiveness, and consequently to provide recommendations for future research.

\section{METHODS}

\section{Data compilation}

To identify studies reporting trait differences between invasive and non invasive plant species measured in greenhouse or garden experiments or in field sites where the invasive and non invasive species co occur, we searched BIOSIS Previews (1986 2008) for the key word combina tion 'native* or non invasive*' and 'alien* or exotic* or invasive*' in the title. From the 931 obtained references, we only kept the ones dealing with plants. We also considered further studies cited in these references, studies published as book chapters and one study published as an MSc thesis.

We ensured by examining the species descriptions in the original studies that the term 'invasive' refers to spreading alien species having an ecological or economic impact, or reaching high local abundance in the region where the study was conducted. Because some studies did not report sample sizes or error terms for mean values per species, not all studies could be used. In our meta analysis, we only included studies that reported traits related to physiology, light interception (i.e. leaf area), shoot allocation (i.e. the inverse of root allocation), growth rate, plant size and fitness (Table 1), because reports on other traits were too scarce.
Furthermore, we only included studies that reported the invaded range (i.e. the target area approach sensu Pyšek et al. 2004), because only few experimental studies assessed traits of invasive alien species and non invasive species in the invader's native range (van Kleunen \& Johnson 2007; van Kleunen et al. 2008). Our final data set included trait differences from 117 studies (see Appendix S1) encompass ing 125 invasive and 196 non invasive plant species. Six of these studies compared invasive alien species to non invasive alien species, mostly related ones. The other 111 studies compared invasive alien species to native species. Surprisingly, the criteria for selection of the native species were frequently not mentioned explicitly. However, implic itly it appears that the most important selection criterion had been that the species co occur (mentioned in 93 studies) and compete with each other (mentioned in 44 studies). Fifty nine studies appear to have used as main or additional criterion that the invasive alien and native species are closely related to each other (i.e. are confamilial).

As the unit of analysis (i.e. the unit for calculation of effect sizes and their variances), we used pairs of invasive and non invasive species. Because studies that included more than one invasive and non invasive species varied largely in the way they reported the data, the pairing of invasive and non invasive species depended on the infor mation available for each study. If the study explicitly assigned species to pairs, we used these species pairs. If the study did not explicitly assign species to pairs, we initially used all potential pairs of invasive and non invasive species within that study. To avoid pseudo replication, we later pooled for each trait per study the effect sizes of the different species pairs per invasive species (see the following section Meta analysis). For each pair of invasive and non invasive species in a study, we calculated, following Rosenberg et al. (2000), from the mean values $(X)$ the effect size (Hedges' $d$ ) as

$d=\frac{\left(\bar{X}_{\text {invasive }}-\bar{X}_{\text {non invasive }}\right)}{S} J$.

Here $S$ is the pooled standard deviation and $J$, which is a weighting factor based on the number of replicates $(N)$ per treatment for each species, was calculated as

$J=1-\frac{3}{4\left(N_{\text {invasive }}+N_{\text {non invasive }}-2\right)-1}$.

A positive value of Hedges' $d$ means that the invasive spe cies had a larger value than the non invasive species. The variance of Hedges' $d$ was calculated as

$v_{d}=\frac{N_{\text {invasive }}+N_{\text {non invasive }}}{N_{\text {invasive }} N_{\text {non invasive }}}+\frac{d^{2}}{2\left(N_{\text {invasive }}+N_{\text {non invasive }}\right)}$.

Hedges' $d$ is the preferred measure of effect size for tradi tional meta analysis, because it has lower Type I error rates 
than other measures, such as the $\log$ response ratio (Lajeunesse \& Forbes 2003). When a study included more than one environment (e.g. treatment or field site), we ini tially calculated effect sizes and their variances for each environment separately. Later we pooled effect sizes of the different environments per species comparison to avoid pseudo replication (for details on pooling see the following section Meta analysis). When a low value of a trait biologi cally indicates the same as high values of other traits in the same trait category (e.g. a low root weight ratio indicates a high shoot allocation), we changed the sign of the effect sizes of this trait accordingly (i.e. we used reversal markers; Table 1).

For each species pair, we extracted from the manuscript data on whether the invasive species is native to Europe, whether the non invasive control species is a native species or a non invasive alien species, whether the study region has a temperate or a subtropical to tropical climate, whether the two species belong to the same family, and whether the two species have the same growth form (annual, herbaceous perennial, woody perennial, succulent, vine, liana and fern). Furthermore, if the non invasive control species was a native species, we also assessed, using external data sources (Randall 2002; Weber 2003), whether the native species is known to be invasive elsewhere.

\section{Meta-analysis}

All meta analytical calculations and statistical comparisons were carried out with the software MetaWin, version 2.1 (Rosenberg et al. 2000). Some studies reported for a species pair more than one trait of a trait category (Table 1), used several environments or used the same invasive alien species in several species pairs (i.e. compared one invasive alien species to more than one non invasive species). To avoid pseudo replication, we pooled effect sizes and variances for each trait category per invasive alien species in a study by doing a separate meta analysis on all traits and environments of the respective trait category. The estimated pooled mean effect size and the mean variance were used in the final data set (see Leimu et al. 2006 for another example of this approach). An alternative approach would be to randomly select a single effect size of a trait category per invasive species per study, but this would lower the information content of the analysis. For pooling of effect sizes and all analyses, we used the so called random model setting (i.e. we used mixed effects meta analysis models), which implies that we assumed that differences among pairs of species and among studies are not only due to sampling error but also due to true random variation, as is the rule for ecological data (Gurevitch \& Hedges 2001).

To test whether mean effect sizes differed significantly from zero, we assessed whether the bias corrected 95\% bootstrap confidence intervals based on 4999 permutations did not include zero (Adams $e t$ al. 1997). For each of the six trait categories separately, we tested with a chi squared test whether heterogeneity among effect sizes of the individual species comparisons $\left(Q_{\text {total }}\right)$ was significantly larger than the expected sampling error. Then, to test whether mean effect sizes of trait categories differed between the levels of factors (see below), we assessed the significance of the between group heterogeneity $\left(Q_{b}\right)$ with a randomization test (Adams et al. 1997). We also tested with a chi squared test whether the remaining within group heterogeneity $\left(Q_{w}\right)$ was significant.

We tested whether mean effect sizes differed among trait categories (Table 1). For each trait category, we also tested whether mean effect sizes differed between species com parisons in which the non invasive control species is a native species and the ones in which the non invasive control species is an alien species. For each of the invasive alien vs. native comparisons, we tested whether mean effect sizes differed between species comparisons in which the native control species is known to be invasive elsewhere and the ones in which the native control species is not known to be invasive elsewhere. We also tested whether mean effect sizes differed between confamilial and non confamilial species comparisons, and between species comparisons with the same growth form and the ones with different growth forms. Furthermore, we tested whether mean effect sizes differed between temperate and subtropical or tropical climatic regions, and whether mean effect sizes differed between species comparisons of which the invasive species is native to Europe and the ones of which the invasive species is not native to Europe. Finally, we tested whether mean effect sizes depended on the quality of the test environment. To this aim, we only included the subset of 73 studies that assessed traits in more than one environment. We included effect sizes for the benign environments, defined as the environments in which the plants had the highest average performance, and the stress environments, defined as the environments in which the plants had the lowest average performance.

Standardized effect sizes of the raw data were slightly (Spearman $r \quad$-0.103), but significantly ( $P$ 0.039), neg atively associated with sample size. This might suggest that studies with small sample sizes are slightly more likely to be published when they found higher trait values for invasive than non invasive species (Rosenberg et al. 2000). On the other hand, a plot of the effect sizes against the sample size revealed a funnel shaped distribution of the data points (Appendix S2), as would be expected in the absence of a sampling bias (Light \& Pillemer 1984; Palmer 1999). Moreover, a plot of the standardized effect sizes against the normal quantiles revealed a straight line, indicating that the effect sizes are normally distributed (Wang \& Bushman 
1998). Overall, this indicates that there is only mild publication bias, and that the effect sizes are normally distributed.

\section{RESULTS}

Averaged over all species comparisons, invasive alien species had higher trait values than non invasive species as indicated by the fact that mean effect sizes of all six trait categories were significantly larger than zero (Fig. 1a). The magnitude of differences between invasive alien species and non invasive species did not differ significantly among the trait categories (Fig. $1 \mathrm{a} ; Q_{\mathrm{b}} \quad 2.76, P \quad$ 0.882). There was also significant heterogeneity in effect sizes within trait categories $\left(Q_{\text {within }} \quad 714.08\right.$, d.f. $\left.\quad 334, P<0.0001\right)$. In line with this, also for each of the six trait categories separately, heterogeneity among effect sizes of the individual species comparisons was significantly larger than the expected sampling error $\left(Q_{\text {total }}\right.$ ranged from 96.39 for 'leaf area allocation' to 145.38 for 'size', all $P<0.0001)$. These results indicate that some of the heterogeneity in effect sizes among species comparisons might be explained by design related or biogeographical and biological factors.

\section{Effects of factors related to the design of studies on trait differences}

For comparisons of invasive alien species to native species, mean effect sizes of all trait categories but 'leaf area allocation' were significantly larger than zero (Fig. 1b). For comparisons of invasive alien species to non invasive alien species, mean effect sizes were also significantly larger than zero for 'size' and 'fitness', but the positive mean effect sizes of the other trait categories were not significant (Fig. 1b). However, a direct comparison of mean effect sizes between the two types of comparisons did not reveal significant differences (Fig. 1b; Appendix S3). These results indicate that invasive species have higher values than native species for traits associated with high performance, and that overall there is a similar pattern for invasive species compared to non invasive alien species.

For comparisons of invasive alien species to native species that are not known to be invasive elsewhere, mean effect sizes of all trait categories but 'leaf area allocation' were significantly larger than zero (Fig. 1c). On the other hand, for comparisons of invasive alien species to native species that are known to be invasive elsewhere, i.e. when comparing invasives in their invasive range with invasives in their native range, none of the mean effect sizes was significantly larger than zero (Fig. 1c). Although mean effect sizes were for most trait categories smaller when the native species are known to be invasive elsewhere than when they are not, a direct comparison of mean effect sizes between the two types of comparisons did not reveal significant differences (Fig. 1c; Appendix S3). Nevertheless, these results suggest that invasive alien species may not have trait values different from the ones of native species that are invasive elsewhere themselves.

For confamilial comparisons, mean effect sizes were significantly larger than zero for all trait categories but 'physiology' and 'leaf area allocation' (Fig. 1d). For non confamilial comparisons, mean effect sizes were significantly larger than zero for the trait categories 'physiology' and 'size' (Fig. 1d). However, a direct comparison of mean effect sizes between the two types of comparisons did not reveal significant differences between confamilial and non confa milial comparisons (Fig. 1d; Appendix S3). Overall, these results suggest that trait differences between invasive and non invasive species could depend on whether the study corrects for phylogeny.

For comparisons between species of the same growth form, mean effect sizes of all trait categories but 'leaf area allocation' were significantly larger than zero (Fig. 1e). For comparisons between species of different growth forms, mean effect sizes of all trait categories but "leaf area allocation' and 'shoot allocation' were significantly larger than zero (Fig. 1e). Moreover, the mean effect size of the trait category 'growth rate' was significantly smaller for comparisons between species with the same growth form than for comparisons between species with different growth forms (Fig. 1e; Appendix S3). These results suggest that, at least for studies comparing growth rates between invasive and non invasive species, it is important to correct for growth form.

\section{Effects of biogeographical and biological factors on trait differences}

For comparisons in temperate regions, mean effect sizes of all trait categories but 'leaf area allocation' and 'fitness' were significantly larger than zero (Fig. 1f). For comparisons in tropical or subtropical regions, mean effect sizes of all trait categories but 'leaf area allocation', 'shoot allocation' and 'size' were significantly larger than zero (Fig. 1f). A direct comparison of mean effect sizes between the two types of comparisons showed that mean effect sizes of the trait categories 'physiology' and 'growth rate' were significantly larger when studies were performed in tropical or subtrop ical regions than when they were performed in temperate regions (Fig. 1f; Appendix S3). These results indicate that the magnitude of trait differences can strongly depend on the climatic region.

For comparisons including invasive alien species from Europe, mean effect sizes of all trait categories but 'leaf area allocation' and 'size' were significantly larger than zero (Fig. 1g). For comparisons including invasive alien species 


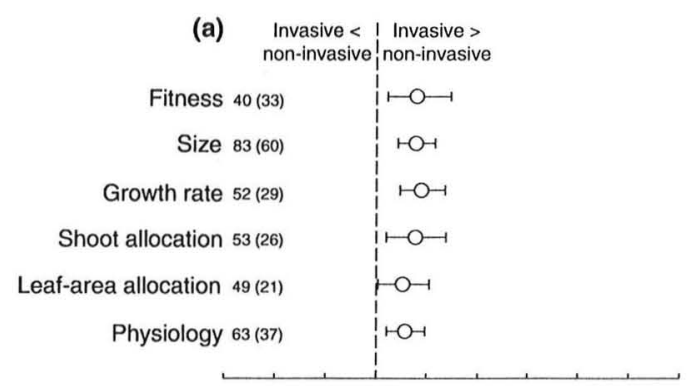

(c)

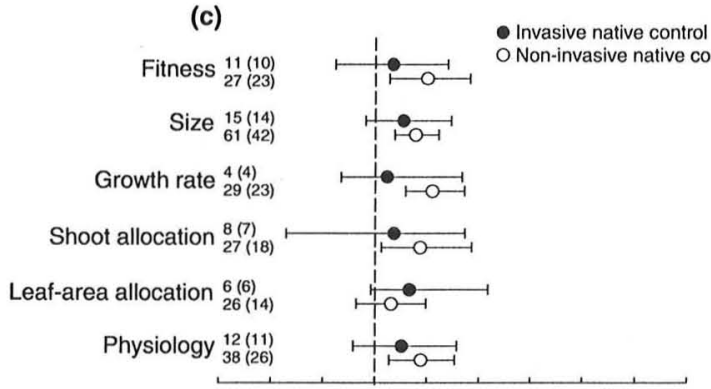

(e)

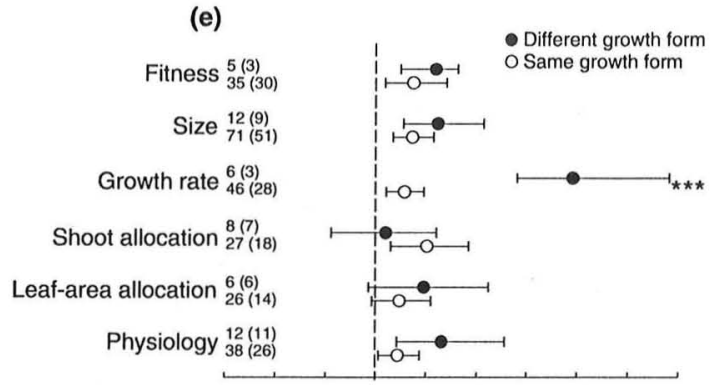

(g)

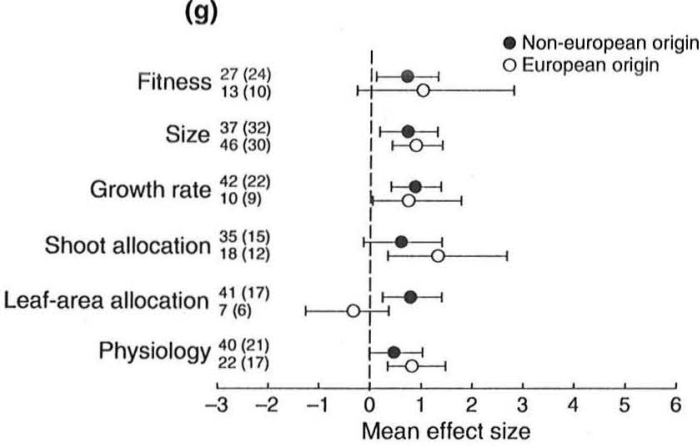

(b)

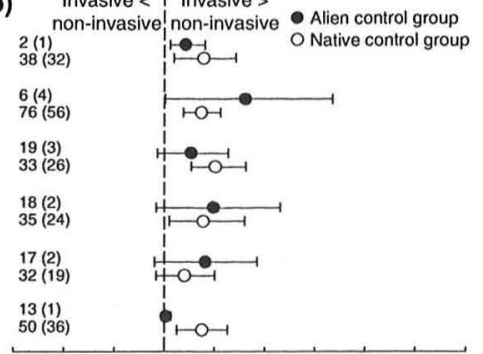

(d)

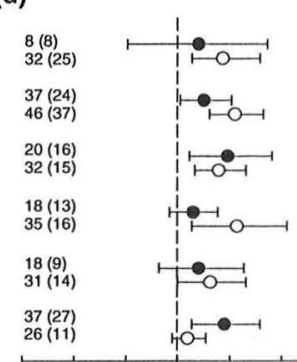

- Non-confamilia O Confamilial (f)

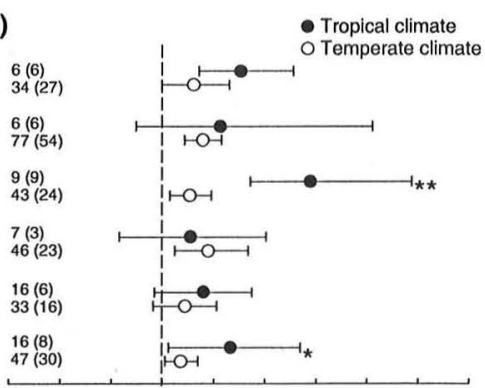

(h)

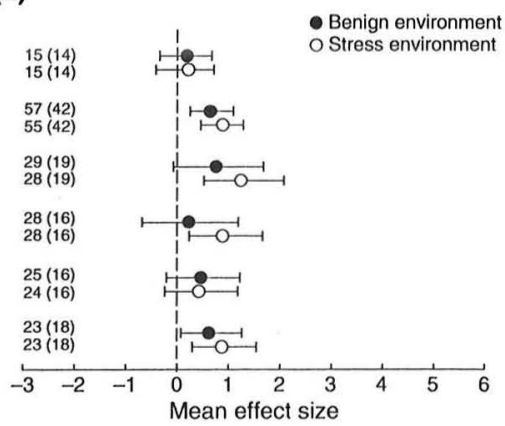

Figure 1 Mean effect sizes (Hedges' $d$ ) of differences between invasive alien plant species and non invasive plant species for (a) the six trait categories (Table 1), and the dependency of these mean effect sizes on (b) whether the control species was a non invasive alien species or a native species, (c) whether the native control species is known to be invasive elsewhere, (d) whether the invasive alien species and non invasive species belong to the same family, (e) whether the invasive alien species and non invasive species have the same growth form, (f) whether the study was performed in a temperate region or in a (sub)tropical region, (g) whether the invasive alien species is native to Europe and $(\mathrm{h})$ whether the species were compared under benign environmental conditions or under more stressful environmental conditions. The bars around the means denote bias corrected $95 \%$ bootstrap confidence intervals. A mean effect size is significantly different from zero when its $95 \%$ confidence interval does not include zero. The sample sizes (i.e. number of species comparisons) and, in parentheses, the numbers of studies are given on the left hand side of each graph. Positive mean effect sizes indicate that the invasive alien species had larger trait values than the non invasive species. Significant differences between factor levels (see Appendix S3): $* P<0.05 ; * * P<0.01 ; * * * P<0.001$. 
from other continents, mean effect sizes of all trait categories but 'physiology' and 'shoot allocation' were significantly larger than zero (Fig. 1g). A direct comparison of mean effect sizes between the two types of comparisons did not reveal significant differences (Fig. 1g; Appendix S3). These results indicate that the invasive species native to Europe do not differ largely in their traits from invasive species native to other continents.

Sixty seven studies included more than one test environ ment. For comparisons in the most stressful environment, mean effect sizes of all trait categories but "leaf area allocation' and 'fitness' were significantly larger than zero (Fig. 1h). For comparisons in the most benign environment, only mean effect sizes of the trait categories 'physiology' and 'size' were significantly larger than zero (Fig. 1h). However, a direct comparison of mean effect sizes between the two types of comparisons did not reveal significant differences (Fig. 1h; Appendix S3). Therefore, overall these results suggest that trait differences between invasive and non invasive species do not depend on the test environment.

\section{DISCUSSION}

The question of what kinds of traits promote plant invasiveness is of utmost significance for an understanding of plant success in general and particularly for understand ing the mechanisms of alien plant invasions. For managing plant invasions and taking preventive measures against them, recognizing invasive species based on functional traits constitutes an indispensable cornerstone of designing risk assessment protocols (Groves et al. 2001). Finding such functional traits with general validity is best achieved by analysing existing comparative studies, e.g. by meta analysis.

Our meta analysis demonstrated clear differences between invasive and non invasive species in traits related to physiology, leaf area allocation, shoot allocation, growth rate, size and fitness. This indicates that invasive species have higher values than non invasive species for traits associated with high performance. It is likely that we found more trait differences between invasive and non invasive plant species than previous reviews (Daehler 2003; Pyšek \& Richardson 2007), because meta analysis is a more powerful approach than simply counting the number of significant and non significant differences between invasive and non invasive species. On the other hand, another recent meta analysis found that invasive alien species did not differ significantly from congeneric native species in size (also including growth rate in that study), and that invasive alien species even had a lower allocation to reproduction (i.e. fitness) than native species (Hawkes 2007). This discrepancy between the results of Hawkes (2007), based on 15 studies, and our results also remained when we restricted our analysis to the 72 studies using confamilial comparisons.
This emphasizes the importance of including many studies in meta analysis to achieve sufficient statistical power.

The consistency of differences between invasive and non invasive species across the different trait categories could reflect that many traits vary in concert, either due to trade offs or because some traits are composite traits (Grotkopp et al. 2002; Westoby et al. 2002). For example, photosyn thetic capacity per unit leaf mass (trait category 'physiology') is frequently positively associated with specific leaf area (trait category 'leaf area allocation'; Wright et al. 2004; Leishman et al. 2007), which is frequently positively associated with high relative growth rates (trait category 'growth rate'; Lambers \& Poorter 1992; Grotkopp et al. 2002). High growth rates in turn are likely to result in high annual biomass production (trait category 'size') and seed produc tion (trait category 'fitness'). Therefore, our finding that invasive species have higher values than non invasive species for each of the trait categories is in line with recent findings that many invasive plants are, in contrast to the majority of coexisting native plants, at a position along the global multi trait leaf economics spectrum that favours fast growth (Leishman et al. 2007). A challenge for future research will be to asses which traits directly confer invasiveness, and which traits are just correlated with those.

Although our analysis revealed clear trait differences between invasive and non invasive species, such differences could also partly reflect potential bias towards studying those invasive species being very apparent as a consequence of their high performance or a bias towards publication of studies that found higher trait values for invasive than for non invasive species. As mentioned in the Methods section, standardized effect sizes were slightly negatively associated with sample size indicating that particularly studies with small sample sizes are slightly more likely to be published when they found higher trait values for invasive than non invasive species. However, this publication bias was very mild (also see funnel plot in Appendix S2). Moreover, there is no reason to believe that publication bias would affect our tests of the effects of biological and experimental factors on trait differences between invasive and non invasive species.

\section{Effects of type of study on trait differences}

Multi species comparative studies that do not include traits measured under common environmental conditions, but use information compiled in floral compendia or other data bases, most frequently compared invasive alien species with non invasive alien species (Kolar \& Lodge 2001; Pyšek \& Richardson 2007; Hayes \& Barry 2008). In contrast, most of the studies that measured species traits under common environmental conditions compared invasive alien species with native species (our study and Pyšek \& Richardson 2007). Most likely this bias reflects the fact that it is easier to 
find plant material for experimentation of comparable native species than to find plant material of comparable non invasive alien species in the non native range (Pyšek \& Richardson 2007). It may also reflect that experimental studies often aim at understanding the invasion of a particular species at a particular local field site, representing a situation in which it is important to know whether the invader successfully competes with natives.

The invasive alien vs. native comparisons and invasive alien vs. non invasive alien comparisons both test for species traits associated with invasiveness but their exact interpretations are different (Hamilton et al. 2005). Mean effect sizes of invasive alien vs. native comparisons were significantly larger than zero for all trait categories with the exception of 'leaf area allocation', whereas mean effect sizes of invasive alien vs. non invasive alien comparisons were only significantly different from zero for 'size' and 'fitness'. Although none of the differences between invasive alien vs. native comparisons and invasive alien vs. non invasive alien comparisons was statistically significant, this suggests that the traits that provide invasive alien species with an advantage over native species are not necessarily the same as the traits that determine whether an alien species will become invasive or not. Whereas the latter is related to the establishment of an alien species, the former is related to the invasion process. For the time being, the discrepancy between the two types of comparisons might also partly reflect the low statistical power for the invasive alien vs. non invasive alien comparisons due to the limited number of studies using this comparative approach (six out of 117 studies). Indeed, none of the mean effect sizes was significantly different between the two types of compari sons. Therefore, we urgently need more studies comparing invasive alien species to non invasive alien species.

One potential problem with invasive vs. native compar isons is that many of the native species might also be successful (i.e. considered 'invasive') in their native range or may even be invasive elsewhere, which would imply that some studies compared invasive with invasive species (Rejmánek 1999; Muth \& Pigliucci 2006). There were no significant differences in mean effect sizes of any of the six trait categories between comparisons that used native species known to be invasive elsewhere and comparisons that used native species not known to be invasive elsewhere. Nevertheless, while mean effect sizes for all trait categories but 'leaf area allocation' were significantly larger than zero when the native species are not known to be invasive elsewhere, mean effect sizes were closer to zero and not significantly different from zero when the native species is known to be invasive elsewhere. This indicates that including comparisons of invasive alien species to native species that are themselves not invasive elsewhere increases the power of detecting traits consistently associated with invasiveness. Therefore, future studies comparing invasive alien species to native species should take care that the native species are not invasive elsewhere in the world.

As a consequence of a shared phylogenetic history, species may not be considered independent data points (Felsenstein 1985). Therefore, studies comparing invasive and non invasive species should account for phylogeny. When we restricted our analysis to confamilial comparisons, mean effect sizes of all trait categories but 'physiology' and 'leaf area allocation' were significantly larger than zero (Fig. 1d). This suggests that most trait differences between invasive and non invasive species are robust with respect to phylogeny.

In addition to phylogenetic or taxonomic non indepen dence between invasive and non invasive species, it is also possible that there is such non independence among effect sizes of species pairs. Recently, Adams (2008) and Lajeu nesse (2009) proposed methods of phylogenetic meta analysis that can account for phylogenetic non indepen dence among effect sizes. These methods, however, cannot account for the phylogeny of both species in a pair (Morales \& Traveset 2009). Therefore, phylogenetic meta analysis is not an appropriate analysis option yet for the kind of data that we have. However, to test whether there is some taxonomic pattern in the magnitude of the effect sizes, we tested whether mean effect sizes differed among taxonomic classes, i.e. between ferns, monocotyledons and the two classes of dicotyledons. For none of the six trait categories was there any evidence that mean effect sizes differed between taxonomic classes (Appendix S4). Therefore, we conclude that it is likely that our results can be generalized across taxonomic groups.

The mean effect size of 'growth rate' was smaller when paired species had the same growth form than when they had different growth forms. Nevertheless, even for com parisons of species with the same growth form, the mean effect size of 'growth rate' was significantly larger than zero, indicating that the association of fast growth with invasive ness is robust. On the other hand, differences between invasive alien plant species and non invasive plant species for the trait categories 'shoot allocation' and 'fitness' became only apparent when confamilial species were compared, while the reverse was true for 'physiology'. This indicates that studies that do not account for phylogeny might fail to detect traits associated with invasiveness or do detect traits that are spuriously associated with invasiveness.

\section{Effects of biogeographical and biological factors on trait differences}

It is unlikely that exactly the same sets of traits distinguish invasive alien species from non invasive species in all parts of the world, and particularly so in regions with different 
climates (Pyšek \& Richardson 2006). Nevertheless, the overall direction of trait differences between invasive alien species and non invasive species was the same for species in temperate regions and species in tropical or subtropical regions. However, the mean effect sizes for 'physiology' and 'growth rate' were significantly larger for species compar isons in tropical or subtropical regions than for species comparisons in temperate regions. This could indicate that in tropical settings specific physiological characteristics and especially fast growth rates are required to allow alien species to invade there. The number of species comparisons in the tropics was, however, relatively low, and it remains to be seen whether these differences remain when more species would be compared in the tropics.

It has been suggested that the high proportion of invasive plant species with European origin among globally invasive species is a consequence of their long history in anthropo genic environments (e.g. Gray 1879; La Sorte et al. 2007). Selection in these environments may have resulted in the evolution of traits that increase invasiveness elsewhere of these European plant species. Among the six trait categories in our meta analysis, none of the mean effect sizes was significantly larger when the comparison included an invasive species from Europe instead of one from other continents. This indicates that although many European species may have functional traits that increase invasiveness, the non European species that have become invasive have largely the same functional traits as invasive species from Europe.

Plant species might differ in phenotypic plasticity, and as a consequence trait differences between species might depend on the environment (Richards et al. 2006). Indeed, Daehler (2003) reported that differences between invasive alien plant species and native plant species strongly depended on the environment, and that the performance of the native species was equal or superior to that of the invasive alien species under low levels of resources. This suggests that invasive alien species might be better capable of taking advantage of the high resource availability in benign environments (Richards et al. 2006), as frequently found after disturbance of habitats (Davis et al. 2000). However, our meta analysis showed that mean effect sizes of the different trait categories did not depend on the quality of the environment. So, although for individual compari sons trait differences between invasive alien species and non invasive species might depend on the environment, averaged over all comparisons trait differences between invasive alien species and non invasive species are robust across environments.

\section{Conclusions and recommendations for future research}

Due to scepticism about the existence of traits generally conferring invasiveness it has been suggested that invasions are fully idiosyncratic (Crawley 1987; Roy 1990). Neverthe less, the number of studies that measured traits of both invasive alien plant species and non invasive plant species under common environmental conditions has steadily increased over the last 30 years (Pyšek \& Richardson 2007). Combining the results of these studies in a single meta analysis enabled us to show that invasive plant species generally have higher values of performance related traits characterizing physiology, leaf area allocation, shoot alloca tion, growth rate, size and fitness than non invasive plant species do. The most important conclusion from these findings is that there are traits promoting invasiveness under many different circumstances.

Despite a steady increase, the number of studies comparing traits of invasive and non invasive species grown under common environmental conditions is still relatively low and very heterogeneous with respect to the type of traits measured and the design of the study. As a consequence, the statistical power to detect mean effect sizes significantly different from zero for subsets of the data was still limited (Fig. 1). Moreover, our meta analysis was restricted to testing effects of those factors on trait differences between invasive and non invasive species for which we had sufficient data. In all our analyses, the heterogeneity in effect sizes that remained unexplained was considerable as indicated by significant $Q_{w}$ values (Appendix S3). Even when we tried to reduce heterogeneity by doing a meta analysis on the subset of data that included only comparisons between invasive and native species that are confamilial and have the same growth form, and for which the native species is not known to be invasive elsewhere, $Q_{w}$ was still significant (Appendix S5). One potential cause of heterogeneity in effect sizes could be that in some studies non invasive species might have been misclassified due to limited knowledge on their behaviour. The heterogeneity in effect sizes also indicates that further explanatory variables need to be investigated. However, many other interesting questions, such as whether different traits are important for invasiveness in different habitat types, particularly undis turbed natural habitats and highly disturbed anthropogenic habitats, can only be addressed once more data become available. Therefore, we recommend that future studies should address explicitly the question whether traits associated with invasiveness differ between habitat types.

Most studies included only one or a few species pairs, and each of these studies consequently had a low number of replicates and thus a low weight in the analyses. A notable exception is a study of Grotkopp \& Rejmánek (2007), which measured seedling growth rates of 15 invasive alien tree species and 13 non invasive alien tree species. Such experimental multi species comparisons (also see van Kleunen \& Johnson 2007) will considerably increase our power to test which traits are consistently associated with 
invasiveness and under which conditions. Our meta analysis also shows that future studies comparing invasive alien species and non invasive ones should account for growth form. We also recommend that future studies comparing invasive alien species with native species should consider whether the native species itself is invasive elsewhere. Finally, we recommend that new studies should more frequently compare invasive alien species with non invasive alien species rather than only with native species, because this type of comparison has been rare and addresses a question fundamentally different from the one of invasive vs. native comparisons. The question why some alien species are successful whilst others are not is pertinent to designing weed risk assessment protocols. Here, the aim is to differentiate between these two groups of alien species.

Although there remains much scope for further research into species traits conferring invasiveness, our meta analysis shows that invasive alien species had higher values for those traits related to performance than non invasive species. This is encouraging and suggests that considering species traits might contribute to predicting future plant invasions.

\section{ACKNOWLEDGEMENTS}

We thank Roosa Leimu for technical advice with regard to meta analysis, Hansjörg Dietz, Lesley de Falco, Michelle Leishman and William Conner for providing additional data, and Marcel Rejmánek and four anonymous referees for constructive comments that helped to improve the manu script. We apologize to all those authors whose work we may have missed. M.v.K. and M.F. acknowledge funding by the National Centre of Competence in Research Plant Survival.

\section{REFERENCES}

Adams, D.C. (2008). Phylogenetic meta analysis. Evolution, 62, 567 572.

Adams, D.C., Gurevitch, J. \& Rosenberg, M. (1997). Resampling tests for meta analysis of ecological data. Ecology, 78, 12771283.

Bucharova, A. \& van Kleunen, M. (2009). Introduction history and species characteristics partly explain naturalization success of North American woody species in Europe. J. Ecol., 97, 230238.

Colautti, R.I., Grigorovich, I.A. \& MacIsaac, H.J. (2006). Propagule pressure: a null model for biological invasions. Biol. Invasions, 8, 10231037.

Crawley, M.J. (1987). What makes a community invasible? In: Colonization, Succession and Stability (eds Gray, A.J., Crawley, M.J. \& Edwards, P.J.). Blackwell Scientific Publications, Oxford, UK, pp. 429453.

Daehler, C.C. (1998). The taxonomic distribution of invasive angiosperm plants: ecological insights and comparison to agri cultural weeds. Biol. Conserv., 84, 167180.

Daehler, C.C. (2003). Performance comparisons of co occurring native and alien invasive plants: implications for conservation and restoration. Annu. Rev. Ecol. Evol. Syst., 34, 183211.
Davis, M.A., Grime, J.P. \& Thompson, K. (2000). Fluctuating resources in plant communities: a general theory of invasibility. J. Ecol., 88, 528534.

Felsenstein, J. (1985). Phylogenies and the comparative method. Am. Nat., 125, 115.

Gray, A. (1879). The pertinacity and predominance of weeds. $A \mathrm{~m}$. J. Sci. Arts, 18, 161167.

Grotkopp, E. \& Rejmánek, M. (2007). High seedling relative growth rate and specific leaf area are traits of invasive species: phylogenetically independent contrasts of woody angiosperms. Am. J. Bot., 94, 526532.

Grotkopp, E., Rejmánek, M. \& Rost, T.L. (2002). Towards a causal explanation of plant invasiveness: seedling growth and life his tory strategies of 29 pine (Pinus) species. Am. Nat., 159, 396419.

Grotkopp, E., Rejmánek, M., Sanderson, M.J. \& Rost, T.L. (2004). Evolution of genome size in pines (Pinus) and its life history correlates: supertree analyses. Evolution, 58, 17051729.

Groves, R.H., Panetta, F.D. \& Virtue, J.G. (2001). Weed Risk Assessment. CSIRO Publishing, Collingwood, Australia.

Gurevitch, J. \& Hedges, L.V. (2001). Meta analysis: combining the results of independent experiments. In: Design and Analysis of Ecological Experiments (eds Scheiner, S.M. \& Gurevitch, J.). Oxford University Press, Oxford, UK, pp. 347369.

Hamilton, M.A., Murray, B.R., Cadotte, M.W., Hose, G.C., Baker, A.C., Harris, C.J. et al. (2005). Life history correlates of plant invasiveness at regional and continental scales. Ecol. Lett., 8, 10661074.

Hawkes, C.V. (2007). Are invaders moving targets? The generality and persistence of advantages in size, reproduction, and enemy release in invasive plant species with time since introduction. Am. Nat., 170, 832843.

Hayes, K.R. \& Barry, S.C. (2008). Are there any consistent pre dictors of invasion success? Biol. Invas., 10, 483506.

Hedges, L.V. \& Olkin, I. (1985). Statistical Methods for Meta Analysis. Academic Press, San Diego, CA.

van Kleunen, M. \& Johnson, S.D. (2007). South African Iridaceae with rapid and profuse seedling emergence are more likely to become naturalized in other regions. J. Ecol., 95, 674681.

van Kleunen, M. \& Richardson, D.M. (2007). Invasion biology and conservation biology time to join forces to explore the links between species traits and extinction risk and invasiveness. Progr. Pbys. Geogr., 31, 447450.

van Kleunen, M., Johnson, S.D. \& Fischer, M. (2007). Predicting naturalization of southern African Iridaceae in other regions. J. Appl. Eicol., 44, 594603.

van Kleunen, M., Manning, J.C., Pasqualetto, V. \& Johnson, S.D. (2008). Phylogenetically independent associations between autonomous self fertilization and plant invasiveness. Am. Nat., 171, 195201.

Kolar, C.S. \& Lodge, D.M. (2001). Progress in invasion biology: predicting invaders. Trends Ecol. Evol., 16, 199204.

La Sorte, F.A., McKinney, M.L. \& Pyšek, P. (2007). Compositional similarity among urban floras within and across continents: biogeographical consequences of human mediated biotic inter change. Glob. Change Biol., 13, 913921.

Lajeunesse, M.J. (2009). Meta analysis and the comparative phylogenetic method. Am. Nat., 174, 369381.

Lajeunesse, M.J. \& Forbes, M.R. (2003). Variable reporting and quantitative reviews: a comparison of three meta analytical techniques. Ecol. Lett., 6, 448454. 
Lake, J.C. \& Leishman, M.R. (2004). Invasion success of exotic plants in natural ecosystems: the role of disturbance, plant attri butes and freedom from herbivores. Biol. Conserv., 117, 215226.

Lambers, H. \& Poorter, H. (1992). Inherent variation in growth rate between higher plants: a search for physiological causes and ecological consequences. Adv. Ecol. Res., 23, 187261.

Leimu, R., Mutikainen, P., Koricheva, J. \& Fischer, M. (2006). How general are positive relationships between plant population size, fitness and genetic variation? J. Ecol., 94, 942952.

Leishman, M.R., Haslehurst, T., Ares, A. \& Baruch, Z. (2007). Leaf trait relationships of native and invasive plants: community and global scale comparisons. New Phytol., 176, 635643.

Light, R.J. \& Pillemer, D.B. (1984). Summing up: The Science of Reviening Research. Harvard University Press, Cambridge, MA.

Mason, R.A.B., Cooke, J., Moles, A.T. \& Leishman, M.R (2008). Reproductive output of invasive versus native plants. Glob. Ecol. Biogeogr., 17, 633640.

Mooney, H.A., Mack, R.N., McNeely, J.A., Neville, L.E., Schei, P.J. \& Waage, J.K. (2005). Invasive Alien Species: A New Synthesis. Island Press, Washington, DC.

Morales, C.L. \& 'Traveset, A. (2009). A meta analysis of impacts of alien vs. native plants on pollinator visitation and reproductive success of co flowering native plants. Ecol. Lett., 12, 716728.

Muth, N.Z. \& Pigliucci, M. (2006). Traits of invasives reconsidered: phenotypic comparisons of introduced invasive and introduced noninvasive plant species within two closely related clades. $\mathrm{Am}$. J. Bot., 93, 188196.

Palmer, A.R. (1999). Detecting publication bias in meta analysis: a case study of fluctuating asymmetry and sexual selection. $\mathrm{Am}$. Nat., 154, 220233.

Pimentel, D., Zuniga, R. \& Morrison, D. (2005). Update on the environmental and economic costs associated with alien invasive species in the United States. Ecol. Econ., 52, 273288.

Pyšek, P. (1998). Is there a taxonomic pattern to plant invasions? Oikos, 82, 282294.

Pyšek, P. \& Richardson, D.M. (2006). The biogeography of natu ralization in alien plants. J. Biogeogr., 33, 20402050.

Pyšek, P. \& Richardson, D.M. (2007). Traits associated with invasiveness in alien plants: where do we stand? In: Biological Invasions (ed. Nentwig, W.). Springer, New York, pp. 97125.

Pyšek, P., Richardson, D.M. \& Williamson, M. (2004). Predicting and explaining plant invasions through analysis of source area floras: some critical considerations. Divers. Distrib., 10, 179187.

Randall, R.P. (2002). A Global Compendium of Weeds. Richardson, Melbourne, Australia.

Rejmánek, M. (1999). Invasive plant species and invasible ecosys tems. In: Invasive Species and Biodiversity Management (eds Sandlund, O.T., Schei, P.J. \& Viken, A). Kluwer Academic Publishers, Dordrecht, The Netherlands, pp. 79102.

Rejmánek, M., Richardson, D.M., Higgins, S.I., Pitcairn, M.J. \& Grotkopp, E. (2005). Ecology of invasive plants: state of the art. In: Invasive Alien Species: A New Synthesis (eds Mooney, H.A., Mack, R.N., McNeely, J.A., Neville, L.E., Schei, P.J. \& Waage, J.K.). Island Press, Washington, DC, pp. 104161.

Richards, C.L., Bossdorf, O., Muth, N.Z., Gurevitch, J. \& Pigliucci, M. (2006). Jack of all trades, master of some? On the role of phenotypic plasticity in plant invasions. Ecol. Lett., 9, 981993.
Rosenberg, M.S., Adams, D.C. \& Gurevitch, J. (2000). MetaWin. Statistical Software for Meta Analysis. Sinauer Ass. Inc., Sunderland, MA.

Roy, J. (1990). In search of characteristics of plant invaders. In: Biological Invasions in Europe and the Mediterranean Basin (eds di Castri, J., Hansen, A.J. \& Debussche, M.). Kluwer, Dordrecht, The Netherlands, pp. 335352.

Wang, M.C. \& Bushman, B.J. (1998). Using the normal quantile plot to explore meta analytic data sets. Psychol. Methods, 3, 4654.

Weber, E. (2003). Invasive Plant Species of the World: A Reference Guide to Environmental Weeds. CABI Publishing, Wallingford, UK.

Westoby, M., Falster, D.S., Moles, A.T., Vesk, P.A. \& Wright, I.J. (2002). Plant ecological strategies: some leading dimensions of variation between species. Annu. Rev. Ecol. Syst., 33, 125159.

Wright, I.J., Reich, P.B., Westoby, M., Ackerly, D.D., Baruch, Z., Bongers, F. et al. (2004). The worldwide leaf economics spec trum. Nature, 428, 821827

\section{SUPPORTING INFORMATION}

Additional Supporting Information may be found in the online version of this article:

Appendix \$1 References of the 117 studies included in the meta analysis.

Appendix S2 Funnel plot of effect sizes (Hedges' d) of the raw data against the average sample size of the invasive and non invasive species compared.

Appendix S3 Table with heterogeneity $\left(Q_{b}\right)$ and its signifi cance between levels of the different factors of interest for each trait category.

Appendix S4 Table with mean effect sizes and heterogeneity therein among taxonomic classes for each of the six trait categories.

Appendix S5 Meta analysis on subset of data including only pairs of confamilial invasive and non invasive native species of similar growth form.

As a service to our authors and readers, this journal provides supporting information supplied by the authors. Such materials are peer reviewed and may be re organized for online delivery, but are not copy edited or typeset. Technical support issues arising from supporting information (other than missing files) should be addressed to the authors. 